\title{
'Kiowa' Blackberry
}

\section{James N. Moore ${ }^{\mathbf{1}}$ and John R. Clark ${ }^{\mathbf{2}}$ \\ Department of Horticulture, University of Arkansas, Fayetteville, AR 72701}

Additional index words. Rubus, fruit breeding

'Kiowa' is the eighth in a series of erectgrowing, high-quality, productive blackberry (Rubus L. subgenus Rubus Watson) cultivars developed in the breeding program of the Arkansas Agricultural Expt. Station. 'Kiowa' produces larger fruit and has a longer harvest period than previously released Arkansas cultivars.

\section{Origin}

'Kiowa' (tested as Ark. 1380) resulted from a cross of Ark. $791 \times$ Ark. 1058 made in 1980 (Fig. 1). The original plant was selected from a seedling field in 1983 at the Univ. of Arkansas Fruit Substation, Clarksville. Since then, it has been tested at four locations in Arkansas and on experiment stations in several other states.

\section{Description}

'Kiowa' produces thorned canes, with thorn size and density similar to that of 'Shawnee'. Canes are not as erect as those of 'Shawnee', but are self-supporting and require no trellis.

In Arkansas tests, 'Kiowa' generally has been less productive than 'Shawnee', and its productivity has been similar to 'Choctaw' (Table 1). 'Kiowa' appears to be equally adaptable in all sections of Arkansas and has performed well in eastern Texas (John Lipe, personal communication).

A major attribute of 'Kiowa' is its large fruit size. It consistently produces fruit that are $30 \%$ heavier than 'Shawnee' and are nearly double the weight of 'Choctaw' (Table 2). 'Kiowa' produces large fruit throughout the harvest season (Fig. 2).

In spring, budbreak occurs slightly earlier on 'Kiowa' than on 'Shawnee' and 'Choctaw', but 'Kiowa' blooms later than the other cultivars (Table 3). The first picking of 'Kiowa' fruit occurs an average of 3 days later than that of 'Shawnee', and peak harvest occurs $\approx 6$ days

Received for publication 3 Oct. 1995. Accepted for publication 24 Nov. 1995. Published with the approval of the Director, Arkansas Agricultural Expt. Station, manuscript no. 95098. We thank Elbert Baker, Maurus Brown, Gina Fernandez, Effie Gilmore, David Gilmore, Kelly Irvin, Charles Parsons, Paula Watson, and Jack Young for collecting data during the evaluation of 'Kiowa'. The cost of publishing this paper was defrayed in part by the payment of page charges. Under postal regulations, this paper therefore must be hereby marked advertisement solely to indicate this fact.

${ }^{1}$ Distinguished Professor.

${ }^{2}$ Associate Professor later than for 'Shawnee'. The harvest season of 'Kiowa' is long, with fruit ripening over 6

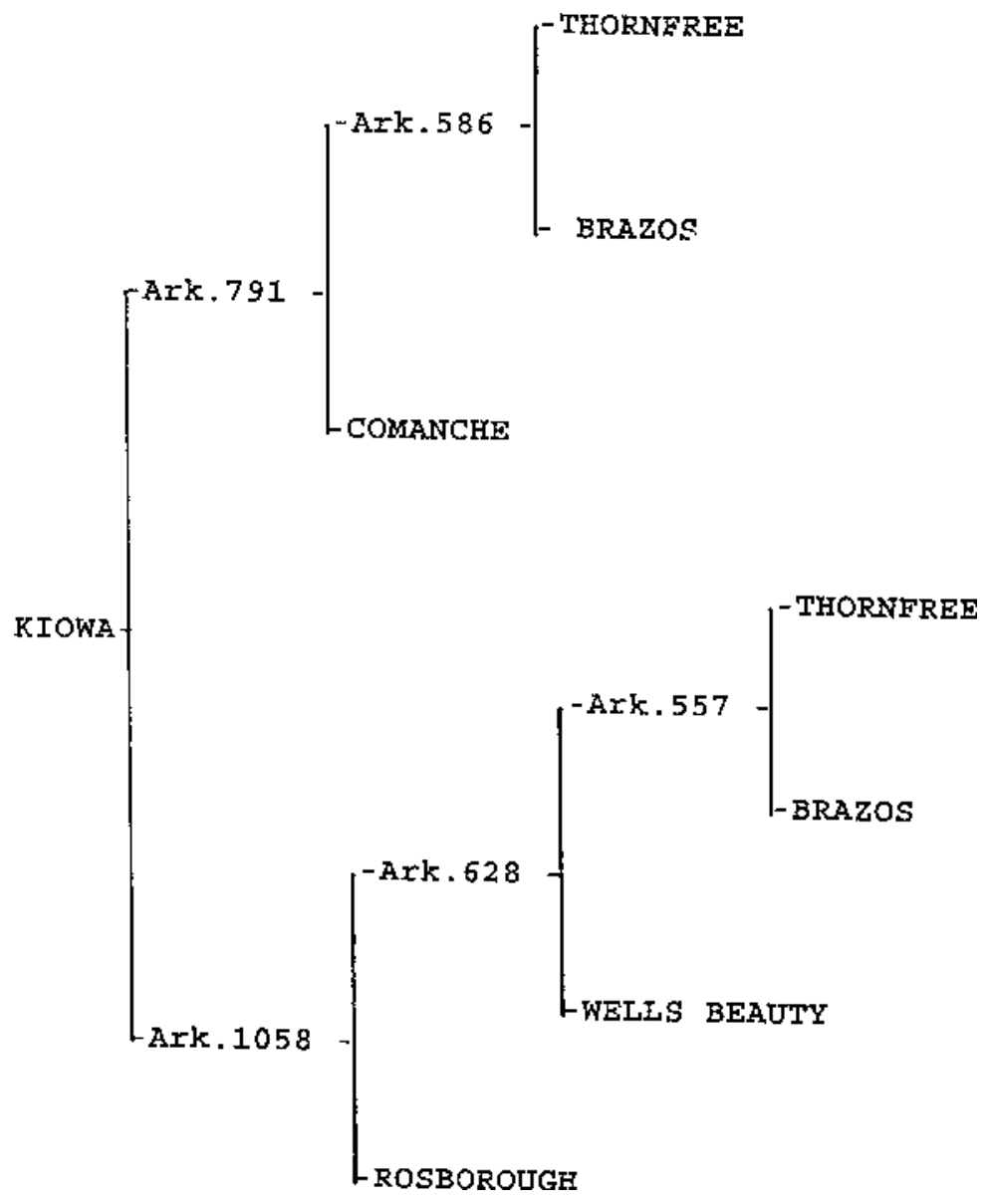

Fig. 1. Pedigree of 'Kiowa' blackberry.

Table 1. Yield (in th ha ${ }^{-1}$ ) of three blackberry cultivars at four locations in Arkansas.

\begin{tabular}{|c|c|c|c|c|c|}
\hline Cultivar & $1990^{2}$ & $1991^{z}$ & $1992^{2}$ & $1993^{y}$ & $1994 \mathrm{y}$ \\
\hline \multicolumn{6}{|c|}{ Clarksville } \\
\hline Kiowa & $4.2 b^{x}$ & $12.6 \mathrm{~b}$ & $12.0 \mathrm{a}$ & $7.2 \mathrm{a}$ & $7.6 \mathrm{~b}$ \\
\hline Shawnee & $9.3 \mathrm{a}$ & $17.0 \mathrm{a}$ & $14.3 \mathrm{a}$ & $7.7 \mathrm{a}$ & $12.3 \mathrm{a}$ \\
\hline Choctaw & $3.0 \mathrm{~b}$ & $13.9 \mathrm{~b}$ & $3.7 \mathrm{~b}$ & $5.1 \mathrm{~b}$ & $6.9 \mathrm{~b}$ \\
\hline \multicolumn{6}{|c|}{ Hope } \\
\hline Kiowa & $1.1 \mathrm{~b}$ & $10.8 \mathrm{~b}$ & $10.9 \mathrm{a}$ & $5.1 \mathrm{~b}$ & $12.8 \mathrm{~b}$ \\
\hline Shawnee & $5.8 \mathrm{a}$ & $11.8 \mathrm{ab}$ & $10.1 \mathrm{a}$ & $9.7 \mathrm{a}$ & $18.2 \mathrm{a}$ \\
\hline Choctaw & $7.2 \mathrm{a}$ & $16.8 \mathrm{a}$ & $9.0 \mathrm{a}$ & $5.1 \mathrm{~b}$ & $8.5 \mathrm{c}$ \\
\hline \multicolumn{6}{|c|}{ Fayetteville } \\
\hline Kiowa & --- & --- & --- & $3.0 \mathrm{a}$ & $7.3 \mathrm{~b}$ \\
\hline Shawnee & --- & --- & --- & $3.5 \mathrm{a}$ & $15.4 \mathrm{a}$ \\
\hline Choctaw & --- & --- & --- & $4.8 \mathrm{a}$ & $13.9 \mathrm{a}$ \\
\hline \multicolumn{6}{|c|}{ Bald Knob } \\
\hline Kiowa & --- & --- & --- & $7.7 \mathrm{a}$ & $8.0 \mathrm{ab}$ \\
\hline Shawnee & --- & --- & --- & $5.9 \mathrm{ab}$ & $9.7 \mathrm{a}$ \\
\hline Choctaw & --- & --- & --- & $4.2 \mathrm{~b}$ & $6.2 \mathrm{~b}$ \\
\hline
\end{tabular}

${ }^{2}$ Planting established in Apr. 1989.

yPlanting established in Apr. 1992.

'Mean separation within locations within columns by Duncan's multiple range test at $P \leq 0.05$. 
but is not as good as that of 'Navaho' or 'Arapaho' (Penny Perkins-Veazie, personal communication). The fresh fruit flavor rates equal to or superior to the flavor of 'Shawnee'. Soluble solids concentration in 'Kiowa' fruit

is usually higher than for 'Shawnee' or 'Choctaw' fruit (Table 3). Seed size, as determined by average dry seed weight, is similar to that of 'Shawnee' but is much larger than that of 'Choctaw' (Table 3).

Table 2. Fruit weight (in g/fruit) of three blackberry cultivars at four locations in Arkansas.

\begin{tabular}{|c|c|c|c|c|c|}
\hline Cultivar & $1990^{z}$ & $1991^{z}$ & $1992^{z}$ & $1993^{y}$ & $1994^{y}$ \\
\hline \multicolumn{6}{|c|}{ Clarksville } \\
\hline Kiowa & $9.9 \mathrm{a}^{\mathrm{x}}$ & $10.3 \mathrm{a}$ & $9.8 \mathrm{a}$ & $11.1 \mathrm{a}$ & $10.0 \mathrm{a}$ \\
\hline Shawnee & $7.4 \mathrm{~b}$ & $6.7 \mathrm{~b}$ & $7.0 \mathrm{~b}$ & $7.6 \mathrm{~b}$ & $5.9 \mathrm{~b}$ \\
\hline Choctaw & $5.1 \mathrm{c}$ & $4.9 \mathrm{c}$ & $5.3 \mathrm{c}$ & $6.1 \mathrm{c}$ & $4.8 \mathrm{c}$ \\
\hline \multicolumn{6}{|c|}{ Hope } \\
\hline Kiowa & $8.3 \mathrm{a}$ & $9.0 \mathrm{a}$ & $8.1 \mathrm{a}$ & $13.0 \mathrm{a}$ & $10.0 \mathrm{a}$ \\
\hline Shawnee & $6.9 \mathrm{~b}$ & $6.3 \mathrm{~b}$ & $5.2 \mathrm{~b}$ & $9.0 \mathrm{~b}$ & $6.7 \mathrm{~b}$ \\
\hline Choctaw & $5.4 \mathrm{c}$ & $4.4 \mathrm{c}$ & $4.2 \mathrm{c}$ & $4.9 \mathrm{c}$ & $4.2 \mathrm{c}$ \\
\hline \multicolumn{6}{|c|}{ Fayetteville } \\
\hline Kiowa & --- & --- & --- & $9.6 \mathrm{a}$ & $7.0 \mathrm{a}$ \\
\hline Shawnee & --- & --- & --- & $5.9 \mathrm{~b}$ & $5.1 \mathrm{~b}$ \\
\hline Choctaw & --- & --- & --- & $5.8 \mathrm{~b}$ & $3.8 \mathrm{c}$ \\
\hline \multicolumn{6}{|c|}{ Bald Knob } \\
\hline Kiowa & --- & --- & --- & $9.3 \mathrm{a}$ & $6.8 \mathrm{a}$ \\
\hline Shawnee & --- & --- & --- & $5.6 \mathrm{~b}$ & $3.7 \mathrm{~b}$ \\
\hline Choctaw & --- & --- & --- & $4.6 \mathrm{c}$ & $2.7 \mathrm{c}$ \\
\hline
\end{tabular}

${ }^{2}$ Planting established in Apr. 1989.

yPlanting established in Apr. 1992

xMean separation within locations within columns by Duncan's multiple range test at $P \leq 0.05$.
'Kiowa' plants are not as vigorous or erect as 'Shawnee' or 'Choctaw' plants (Table 3). However, fruiting row establishment has been good, using either plants or root cuttings. For best production, primocanes of 'Kiowa' should be tipped in summer when they reach 1.0 to 1.2 $\mathrm{m}$ high, and lateral branches should be pruned to $40 \mathrm{~cm}$ during winter dormancy.

'Kiowa' is moderately resistant to anthracnose [Elsinoë veneta (Burkh.) Jenkins], and there have been no disease problems following a fungicide program consisting of a single application of liquid lime sulfur at budbreak. There has been no orange rust [Gymnoconia nitens (Schwein.) F. Kern \& H.W. Thurston] on 'Kiowa'. The reaction of plants to rosette [Cercosporella rubi (Wint.) Plakidas] has not been determined. Plants have shown no visible injury following field exposure to $-23 \mathrm{C}$, and annual ratings for cold hardiness indicate that 'Kiowa' is as hardy as 'Shawnee' and more hardy than 'Choctaw' (Table 3).

The outstanding characteristics of 'Kiowa' are its large fruit size, which is maintained throughout the harvest season, and its long ripening period. 'Kiowa' will be of value for markets in which large fruit and an extended harvest season are desired. Its fruiting charac-

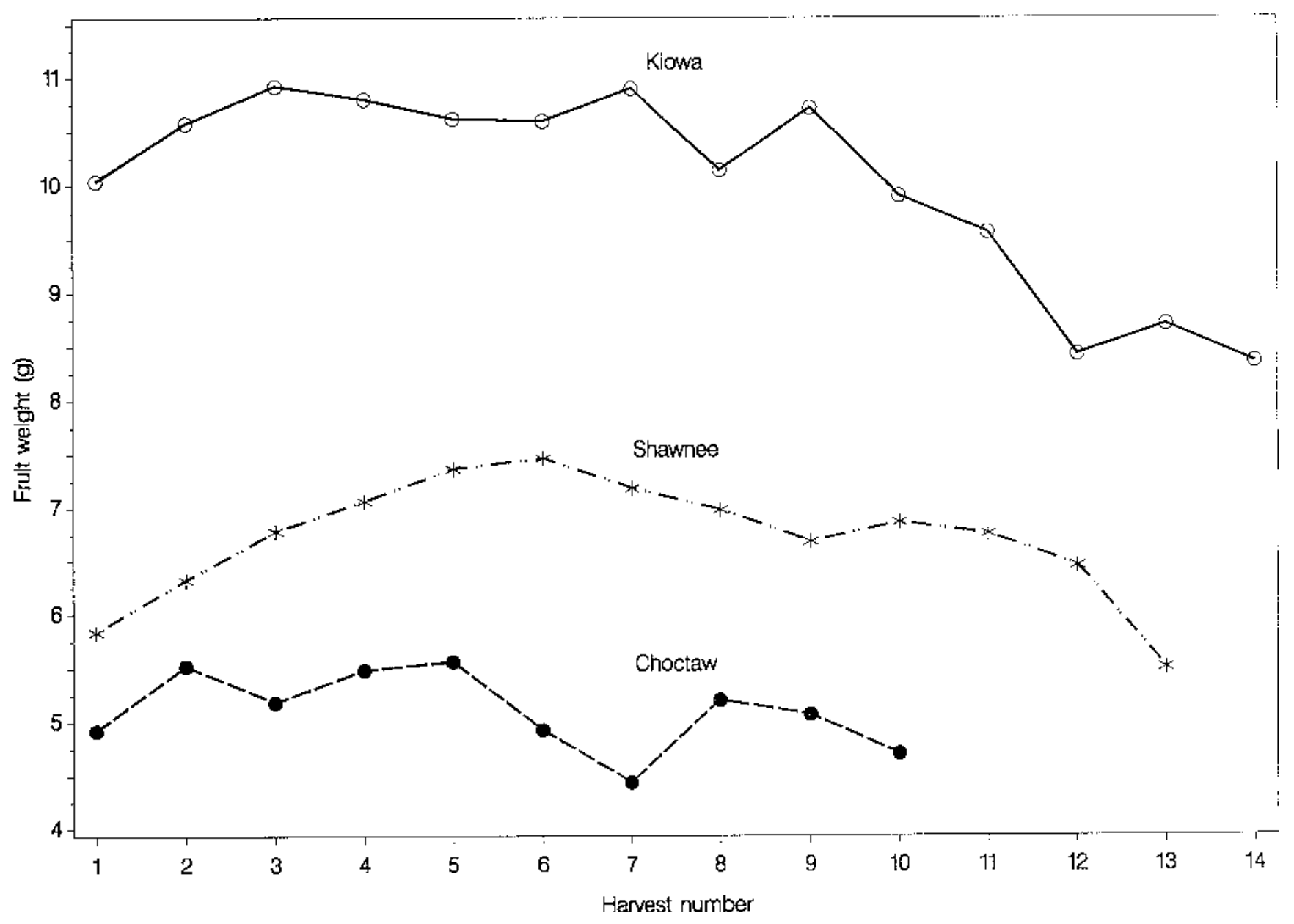

Fig. 2. Fruit weight of three blackberry cultivars at successive harvests at Fruit Substation, Clarksville, Ark. [means of five harvest years (1990 to 1994)]. 


\section{Cultivar \& Germplasm Releases}

Table 3. Plant and fruit characteristics of blackberry cultivars at Fruit Substation, Clarksville, Ark.

\begin{tabular}{|c|c|c|c|}
\hline Characteristic & Kiowa & Shawnee & Choctaw \\
\hline \multicolumn{4}{|l|}{ Date } \\
\hline Budbreak $^{2}$ & 22 Feb. & $25 \mathrm{Feb}$. & 25 Feb. \\
\hline $50 \%$ bloom $^{y}$ & 2 May & $30 \mathrm{Apr}$. & 26 Apr. \\
\hline \multicolumn{4}{|l|}{ Harvest $^{\mathrm{y}}$} \\
\hline First & 12 June & 9 June & 31 May \\
\hline Peak & 30 June & 24 June & 10 June \\
\hline Last & 23 July & 11 July & 22 June \\
\hline \multicolumn{4}{|l|}{ Fruit } \\
\hline Firmness ${ }^{\mathrm{x}, \mathrm{w}}$ & 8.0 & 7.5 & 7.6 \\
\hline Flavor $^{\mathrm{x}, \mathrm{w}}$ & 8.2 & 7.6 & 8.7 \\
\hline Fruit size $^{\mathrm{x}, \mathrm{w}}$ & 10.0 & 8.1 & 7.0 \\
\hline $\begin{array}{l}\text { Seed size } \\
(\mathrm{mg} / \mathrm{seed})^{\mathrm{v}}\end{array}$ & $3.8 \mathrm{a}$ & $3.9 \mathrm{a}$ & $1.9 \mathrm{~b}$ \\
\hline $\operatorname{SSC}(\%)^{\mathrm{y}, \mathrm{u}}$ & 10.3 & 9.9 & 9.2 \\
\hline \multicolumn{4}{|l|}{ Plant } \\
\hline Vigor ${ }^{\mathrm{x}, \mathrm{w}}$ & 7.6 & 9.8 & 9.0 \\
\hline Health & 8.6 & 8.8 & 9.0 \\
\hline Erectness $^{\mathrm{x}, \mathrm{w}}$ & 7.2 & 9.4 & 8.9 \\
\hline Winter injury ${ }^{\mathrm{y}, \mathrm{w}}$ & 9.5 & 9.6 & 8.2 \\
\hline
\end{tabular}

${ }^{2}$ Means of 2 years (1992 and 1994)

${ }^{y}$ Means of 5 years (1990 to 1994).

${ }^{\mathrm{x}}$ Means of 9 years (1986 to 1994).

"Rating scale 1 to 10 , where $10=$ best.

"Data from 1994, mean separation in row by Duncan's multiple range test at $P \leq 0.05$.

"SSC $=$ soluble solids concentration.

teristics also should make 'Kiowa' popular as a home-garden cultivar. 'Kiowa' is expected to perform well in areas in which 'Shawnee' is adapted.

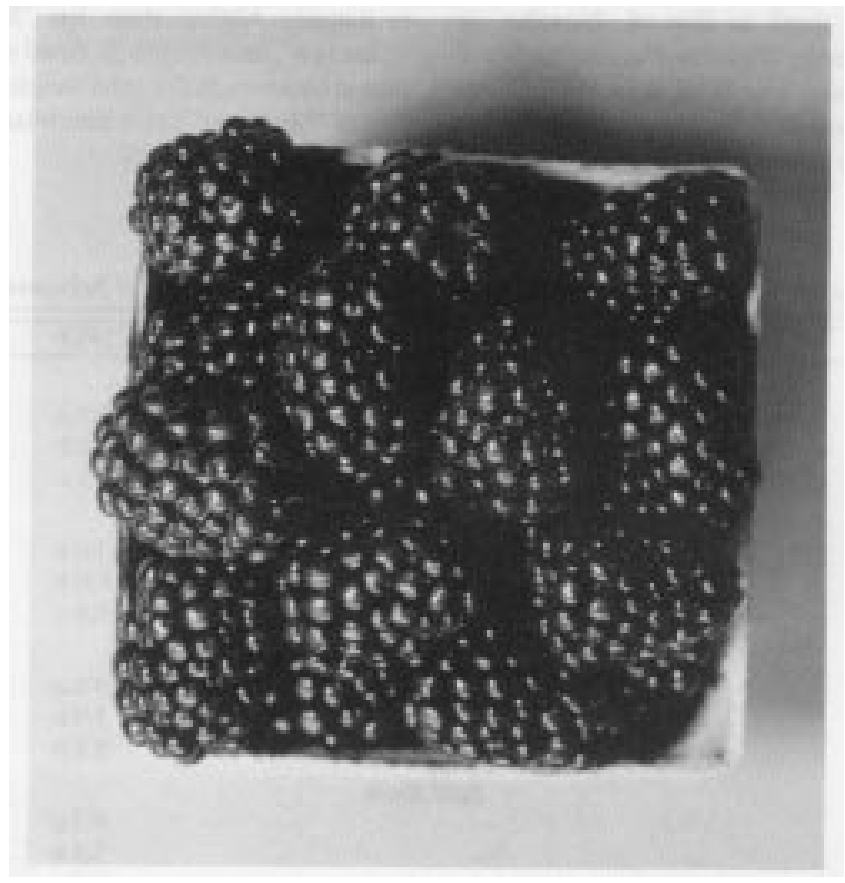

Fig. 3. Fruit of 'Kiowa' blackberry.

\section{Availability}

An application for a U.S. plant patent has been filed for 'Kiowa'. A list of nurseries licensed to propagate and to sell 'Kiowa' can be obtained from J.N.M., 316 Plant Science Building, Univ. of Arkansas, Fayetteville, AR 72701. 